\title{
Cerenkov radiation: a multi-functional approach for biological sciences
}

\author{
Xiaowei Ma ${ }^{1,2}$, Jing Wang ${ }^{2}$ and Zhen Cheng ${ }^{1 *}$ \\ Molecular Imaging Program at Stanford, Bio-X Program and Department of Radiology, Canary Center at Stanford for Cancer Early Detection, Stanford University, \\ Stanford, CA, USA \\ 2 Department of Nuclear Medicine, Xijing Hospital, Fourth Military Medical University, Xi'an, China
}

\section{Edited by:}

David W. Townsend, A* STAR-NUS Clinical Imaging Research Centre, Singapore

\section{Reviewed by:}

Baozhong Shen, The Fourth Hospital of Harbin Medical University, China

Dong S. Lee, Seoul National

University, South Korea

*Correspondence:

Zhen Cheng, Molecular Imaging Program at Stanford, Department of Radiology and Bio-X Program,

Canary Center at Stanford for

Cancer Early Detection, 1201 Welch

Road, Lucas Expansion, P095

Stanford University, Stanford,

CA 94305, USA

e-mail: zcheng@stanford.edu

\section{INTRODUCTION}

Cerenkov radiation (CR) is commonly used in experimental physics to detect small amounts and low concentrations of radioactive atoms. Recently, its applications in biological research have been of great interest. Since 2009, Cerenkov luminescence imaging (CLI), which combines radionuclide labeled probes and optical imaging (OI) instruments, has been actively explored by many research groups. Several recent papers have reviewed the various applications of this new imaging technology [1-6]. Except for CLI, the applications of CR for other purposes, such as radionuclide detection, radiotherapy dose calibration and fluorophores excitation, have also been demonstrated by several groups. CLI was recently also reported for human thyroid imaging, which represents its first use in clinic.

In this review, we will introduce the Cerenkov luminescence (CL) technique, including the basic principle and history of CL. Next, we will discuss some of its basic applications in the past half-century. We will then focus on the preclinical CL studies and the first human use of CLI. In the third and fourth section, we will review some technologies for optimizing CLI and Cerenkov luminescence tomography (CLT). In the last two sections, we will discuss the shortcomings of CLI and some potential directions for technical improvements and biomedical applications.

\section{THE HISTORY AND BASIC THEORY AND FEATURES OF CR}

In 1934, S. I. Vavilov and Pavel Alekseyevich Cerenkov published two reports independently, indicating that high-speed Compton electrons, which were produced by the Compton effect of gamma rays, caused the luminescence of solutions. Subsequently, by further experiments, it was established that the light was produced by charged particles that moved uniformly in the substance at a speed exceeding the phase velocity of light in this medium. This theory was built by Ilya Frank and Igor Tamm in 1936 with the discovery of asymmetry. However, it seems contradictory to the theory of relativity that material bodies are unable to move at the speed of light, much less to exceed it. However, this can be explained by the difference between the speed of the propagation of light waves in a substance and in a vacuum $(c)$, which is caused by the power of the refractive index $(n)$ of the medium. When visible light is transferring in a medium with $n>1$, the speed of the propagation of the light waves in the medium is equal to $c / n$, and consequently, this will be smaller than the velocity of light in a vacuum. For example, taking the refractive index of water to be approximately 1.33 , the speed of light in water is approximately $0.75 \mathrm{c}$. On the other hand, the velocity of the $\beta$ particles emitted from radioactive substances is very near to the velocity of light (c). For ${ }^{18} \mathrm{~F}$, the energy of the positron is $635 \mathrm{keV}$, and according to Einstein's famous relativity equation (Equation 1), the speed of the positron is approximately $0.895 c$.

$$
E=m c^{2}\left(\frac{1}{\sqrt{1-\frac{v^{2}}{c^{2}}}}-1\right)
$$

Thus, according to this principle, these particles can possess a speed that is greater than that of light in this substance $(0.75 c)$.

According to the CR theory, luminescence can only be produced when $\beta n>1$, where $\beta$ is the ratio $v / c$ ( $v$ is the velocity of charged particle and $c$ is the light speed in vacuum). As such, the threshold energy of the charged particles for CR production in 
a medium is relative to the refractive index of the medium, this correlation was figured out by Mitchell et al. [4]. We plotted the threshold energy of charged particles as a function of the refractive index of medium according to the Equation (1) (Figure 1A). For example, the threshold energy of $\beta$ particles in water $(n=$ 1.33 ) is $0.261 \mathrm{MeV}$, while in tissue, assuming a refractive index of 1.4 , it is $0.219 \mathrm{MeV}$.

The equation formulated by J.V. Jelley to calculate the number of photons $(N)$ generated by an electron within a spectral region from $\lambda_{1}$ to $\lambda_{2}$ along an arbitrary distance $L$ is [7]:

$$
N=2 \pi \alpha L\left(\frac{1}{\lambda_{2}}-\frac{1}{\lambda_{1}}\right) \cdot\left(1-\frac{1}{\beta^{2} n^{2}}\right),
$$

where $\alpha$ is the fine structure constant $e^{2} / h c$ equal to $1 / 137$.

In 2010, Spinelli et al. proposed the following equation based on Equation (2) to calculate the total number of light photons, $N_{\text {tot }}$, generated by a positron along an arbitrary distance that is much smaller than the particle range, $L$. [8-10]:

$$
\begin{aligned}
N_{\mathrm{tot}}= & \int_{E_{\min }}^{E_{\max }} g F(Z, E) E^{2}\left(E_{\max }-E\right)^{2} \frac{1}{c} 2 \pi \alpha L \frac{1}{\lambda} \sqrt{1-\frac{m_{0}^{2} c^{4}}{E^{2}}} \\
& \times\left(1-\frac{1}{\left(1-\frac{m_{0}^{2} c^{4}}{E^{2}}\right) n^{2}}\right) d E
\end{aligned}
$$
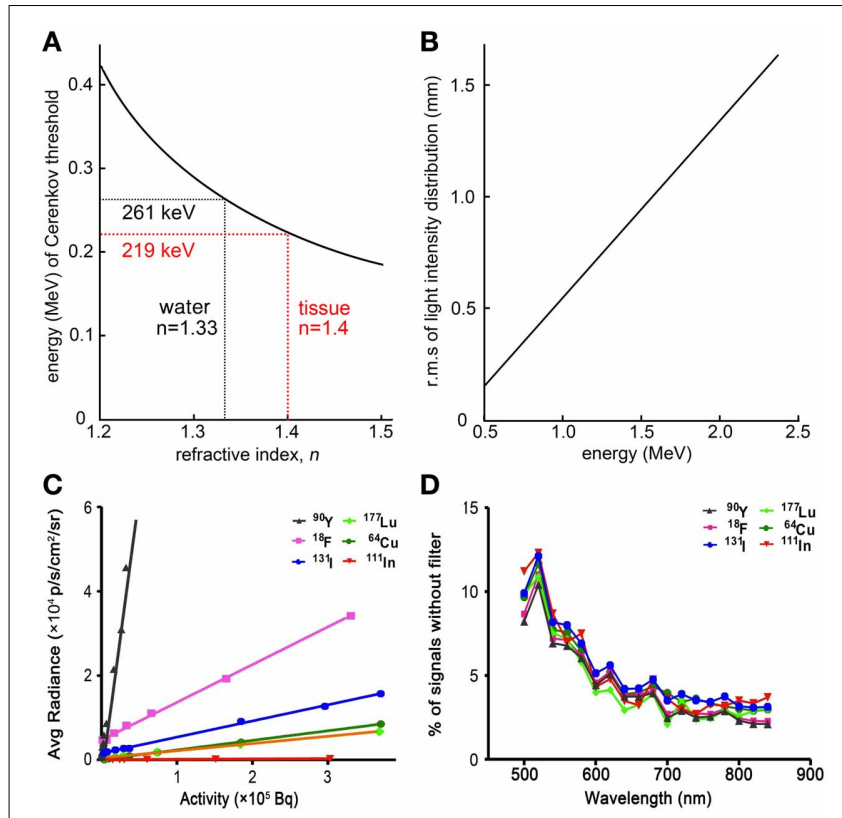

FIGURE 1 | Basic physics of CL. (A) Threshold of charged particles energy for $\mathrm{CL}$ production as a function of refractive index, $n$. (B) The $C L$ intensity distribution as a function of the charged particles energy. (C) Average CL intensity produced by various radionuclides. It showed the $C L$ intensity of all tested radionuclides except for ${ }^{99 \mathrm{~m}} \mathrm{Tc}$ were highly sensitive to the radioactivity. (D) The $\mathrm{CL}$ spectra of various radionuclides. Similar spectra were observed from different radionuclides as the signals of $C L$ will decrease with the increasing of wavelength (C,D were adapted with the permission of Liu et al. [13]).
Accordingly, the Cerenkov luminescence spectrum is equal to the following:

$$
\begin{aligned}
\frac{d N_{\text {tot }}}{d \lambda}= & \alpha_{\text {tot }} \frac{L}{\lambda^{2}} \int_{E_{\min }}^{E_{\max }} F(Z, E) E^{2}\left(E_{\max }-E\right)^{2} \sqrt{1-\frac{m_{0}^{2} c^{4}}{E^{2}}} \\
& \times\left(1-\frac{1}{\left(1-\frac{m_{0}^{2} c^{4}}{E^{2}}\right) n^{2}}\right) d E
\end{aligned}
$$

where $\lambda$ is the wavelength of the CL, $E$ is the minimum positron energy, $E_{\max }$ is the maximum positron energy, $E_{\min }$ is the minimum energy of the particle such that Cerenkov emission takes place, $p$ is the particle momentum, $g$ is a constant, $\alpha_{\text {tot }}$ is given by the following formula:

$$
\alpha_{\text {tot }}=2 \pi \alpha \frac{g}{c}
$$

and $F(Z, E)$ is the Fermi function given by the following formula:

$$
F(Z, E)=-\frac{2 \pi \alpha Z E}{p}\left(1-e^{\frac{2 \pi \alpha Z E}{p}}\right)^{-1}
$$

From the equations above, the CL intensity and distribution correlates with the energy of the charged particles, the refractive index of the medium and the radioactivity of radionuclides.

We plotted the number of CL emitted per millimeter within $400-800 \mathrm{~nm}$ wavelength range as a function of the energy of charged particle according to the Frank-Tamm equation (Figure 1B). It shows that the CL production is highly depends on the energy of particles. And according to the Frank-Tamm equation and the simulation results demonstrated by Mitchell et al., the CL production also relate to the index of refraction when the energy of charged particles is lower than $0.5 \mathrm{MeV}$, and the number of CL (400-800 nm) emitted per decay positively correlate with the energy of charged particles [4]. This means the intensity of CL produced by one radioactive decay is reliable when a certain isotope is used.

Beattie et al. demonstrated a series of quantitative models with some essential corrections to experimentally observe the CL production efficiency and distribution of some common used radionuclides [11]. The models of CR production efficiency were based on the Frank-Tamm equation using several different radionuclides. They showed that the focus of the lens significantly affects the detected sensitivity of CLI. And a more important finding in this work was that the light intensity correlates with the refractive index of the medium. This indicated that the CR should be varieties in different tissues since the refractive index of tissues are very different. But radionuclides emit higher energy charged particles are less sensitive to the variation in refractive index of tissues. As Ackerman et al. reported the CL production is not only dependent on the energy of particles and the refractive index but also on geometry. Hence, different phantoms have been used for quantifying Cerenkov light production in vitro. They found that Cerenkov photon productions were different between the six-well 
plate geometry, simple cubic geometry and centrifuge tube geometry. For the six-well plate simulation, the surface radiance can be considered accurate [12].

The CL intensity also correlates with the radionuclides and radioactivity because the energy of charged particles emitted from different radionuclides are various and the number of charged particles increases with increasing radioactivity of radionuclides. Liu et al. was systemically studied the CL intensity of a variety of radionuclides including $\beta^{-}\left({ }^{90} \mathrm{Y},{ }^{131} \mathrm{I},{ }^{111} \mathrm{In}\right.$ and $\left.{ }^{177} \mathrm{Lu}\right)$, $\beta^{+}\left({ }^{18} \mathrm{~F}\right.$ and $\left.{ }^{64} \mathrm{Cu}\right)$ and $\gamma$-emitters $\left({ }^{99 \mathrm{~m}} \mathrm{Tc}\right)$ (Figure 1C) [13]. As a result, the CL intensity of all tested radionuclides except for ${ }^{99 \mathrm{~m}} \mathrm{Tc}$ were highly sensitive to the radioactivity with 1-5 min OI assay. Because ${ }^{99 \mathrm{~m}} \mathrm{Tc}$ is a pure $\gamma$-emitter with $141 \mathrm{keV} \gamma$-ray, it is hard to produce the CL even through the secondary electrons when the $\gamma$-ray passing through the medium. The results also showed that the spectrum of CL in vitro consistent with the prediction from Equation (3), and it can be seen that the CL spectrum for different radionuclides shared a similar distribution pattern which dependents on $1 / \lambda^{2}$ (Figure 1D). However, the spectrum of ${ }^{18} \mathrm{~F}$-fluorodeoxyglucose $\left({ }^{18} \mathrm{~F}\right.$-FDG) in the heart and bladder of mouse were obviously different with the spectrum of ${ }^{18} \mathrm{~F}-\mathrm{FDG}$ in vitro because the UV and blue band of CL is easily absorbed and scattered by tissue [8].

All of the features of CR indicated that it could be developed qualitatively and quantitatively for radiopharmaceutical and imaging studies in biology since the optical signal is correlated with the activity of radionuclides accumulated in the tissue, the kind of tissue and the depth of the radioactive source. But it also should be normalized with different cameras and distance from the lens to organs. Furthermore, the CL spectrum in different organs is unique, and thus this special wide wavelength of emission spectrum could be used for calculate the depth of the organs or used for CL tomography reconstruction. We will discuss about this in later sections.

\section{APPLICATIONS OF CR}

In 1958, Pavel Alekseyevich Cerenkov shared the Physics Nobel Prize with Ilya Frank and Igor Tamm. In his Nobel Prize lecture, Cerenkov said that "there can be no doubt that the usefulness of this radiation will in the future be rapidly extended" [14]. Indeed $\mathrm{CR}$ has been developed and used in several important applications, such as in charged particle detectors, $\beta$ radionuclide counters, calibrators of clinical electron accelerators and especially, in biological imaging methods. And so far, many studies have theoretically and experimentally evaluated the use of CL with various radionuclides, as shown in Table 1. We will review these important applications of CR in the following sections.

\section{CERENKOV CHARGED PARTICLE DETECTOR}

In the past few decades, even though interest in CR was high, the absence of a sufficiently sensitive and convenient measuring device resulted in CR mainly being used to design detectors for counting charged particles or directly determining the velocity of charged particles. A large number of counters of this sort were described by Jelley [7], Schaumloffel [15], and recently, Arinc [16]. Compared with liquid scintillation methods, the Cerenkov counter is advantageous because of the ease of sample
Table 1 | The properties of radionuclides evaluated for CR applications.

\begin{tabular}{lllcc}
\hline Nuclide & Application & $\begin{array}{l}\text { Decay } \\
\text { mode }\end{array}$ & Energy (keV) & Half-life \\
\hline${ }^{18} \mathrm{~F}$ & PET & $\beta+(97 \%)$ & 633.5 & $109 \mathrm{~min}$ \\
${ }^{13} \mathrm{~N}$ & PET & $\beta+(100 \%)$ & 1198.2 & $9.97 \mathrm{~min}$ \\
${ }^{74} \mathrm{As}$ & PET & $\beta+(26.1 \%)$ & 944.61353 & $17.77 \mathrm{~d}$ \\
& & $\beta-(18.6 \%)$ & & \\
${ }^{64} \mathrm{Cu}$ & PET & $\beta+(17.8 \%)$ & 653 & $12.7 \mathrm{~h}$ \\
${ }^{89} \mathrm{Zr}$ & PET & $\beta+(23 \%)$ & 2400 & $78.4 \mathrm{~h}$ \\
${ }^{124} \mathrm{I}$ & PET & $\beta+(11.7 \%)$ & 1534 & $4.17 \mathrm{~d}$ \\
${ }^{68} \mathrm{Ga}$ & PET & $\beta+(88 \%)$ & 1899 & $67.6 \mathrm{~m}$ \\
${ }^{131} \mathrm{I}$ & SPECT & $\beta-(89.9 \%)$ & 606 & $8.02 \mathrm{~d}$ \\
& & $\gamma(81.7 \%)$ & 364 & \\
${ }^{90} \mathrm{Y}$ & Radiotherapy & $\beta-(99.9 \%)$ & 2280 & $64 \mathrm{~h}$ \\
${ }^{32} \mathrm{P}$ & Radiotherapy & $\beta-(100 \%)$ & 1710 & $14.26 \mathrm{~d}$ \\
${ }^{198} \mathrm{Au}$ & Radiotherapy & $\beta-(98.9 \%)$ & 960.7 & $2.69 \mathrm{~d}$ \\
${ }^{111} \mathrm{In}$ & Radiotherapy & $\beta-(100 \%)$ & 448.3 & $2.8 \mathrm{~d}$ \\
${ }^{177} \mathrm{Lu}$ & Radiotherapy & $\beta-(78.6 \%)$ & 498.3 & $6.73 \mathrm{~d}$ \\
${ }^{225} \mathrm{Ac}$ & Radiotherapy & $\alpha(50.7 \%)$ & 5830 & $10 \mathrm{~d}$ \\
${ }^{* 99 \mathrm{~m}} \mathrm{Tc}$ & SPECT & $\gamma(89 \%)$ & 140.5 & $6.01 \mathrm{~h}$ \\
\hline
\end{tabular}

${ }^{*} 99 m$ Tc has shown to have no significant $C R$.

preparation, the large volume of sample and the easy recoverability of the sample for further chemical treatment. The counting efficiency of Cerenkov counters reach $70 \%$ of ${ }^{32} \mathrm{P}$ disintegration, according to a report by Parker et al. [17]. Although the counting efficiencies of Cerenkov counters are lower than liquid scintillation methods, the use of non-coincidence circuitry, wavelength shifters and plastic vials should increase sensitivity and may permit the extension of the technique to less energetic $\beta$-emitting nuclides [18].

According to a review by Francois in 1973, the Cerenkov detector can be used to assay more than 15 types of $\beta$-emitting radionuclides, including ${ }^{32} \mathrm{P},{ }^{40} \mathrm{~K}$, and ${ }^{198} \mathrm{Au}$ [19]. In their review, the relation between detection efficiency and $\beta$ particle energy was described as a logarithmic function when the maximum particle energy is $0.932-3.55 \mathrm{MeV}$. Subsequent researches have been aimed at optimizing the Cerenkov detector for increased efficiency for radionuclide detection. Recently, Gunther reported a rapid method for determining ${ }^{89} \mathrm{Sr}$ and ${ }^{90} \mathrm{Sr}$ in water and milk samples using Cerenkov counters [20]. ${ }^{210} \mathrm{~Pb}$ also can be measured by combining Cerenkov counters with the established liquid scintillation efficiency tracing technique, according to a report by Arinc et al. [16]. Thus, Cerenkov detector is an important method for measuring $\beta$-emitting radionuclides, especially for pure $\beta$-emitters.

\section{EARLY BIOLOGICAL APPLICATIONS OF CR}

Following the utility of the determination of CR by means of $\beta$-emitters in aqueous solutions, the early use of CR in biological samples was demonstrated by Lanchli in 1969 [21]. They tested the kinetics of the absorption of potassium labeled with ${ }^{86} \mathrm{Rb}$ in excised barley roots at concentrations ranging from 0.01 to $0.2 \mathrm{mM} \mathrm{KCl}$ by determining the CR produced by ${ }^{86} \mathrm{Rb}$. They 
showed that CR could be used successfully to monitor the kinetics of potassium absorption of plants, and inspired by this theory, the CR could also be used to examine the metabolism behavior of other biological materials, such as animal organs or tissues. Two years later, Burch et al. investigated the utility of CR as a diagnostic method to detect the light produced by $0.8 \mathrm{mCi}^{32} \mathrm{P}$, which was injected intravenously (i.v.) into the medial ear vein of an anaesthetized rabbit for eye tumor diagnosis. They found that $\mathrm{CR}$ was a sensitive method for detecting and quantitating radionuclides in tumors [22]. In another study by Smith et al. [23], ${ }^{32} \mathrm{P}$ diisopropyl phosphorofluoridate $\left(\mathrm{DF}^{32} \mathrm{P}, 60-240 \mu \mathrm{Ci}\right)$ was injected into normal and thrombocytopenic dogs via the cephalic vein. The platelets were recovered and processed from 4.5-13.5 mL of blood, and the platelet recovery was calculated by counting the CR.

\section{CERENKOV LUMINESCENCE IMAGING}

In the absence of sufficiently sensitive and convenient imaging devices, CR used to be detected only by a counting detector, as described above. In recent years, advances in the OI devices in biophotonics field have progressed rapidly with the development of highly sensitive, charge-coupled detectors (CCD) that can detect the weak light produced by CR to form a digital image. This new imaging modality was then named as CLI [24].

In 2009, three groups reported the in vivo CL molecular imaging of some common clinically used radionuclides such as ${ }^{18} \mathrm{~F}$ and ${ }^{13} \mathrm{~N}$ for positron emission tomography (PET), ${ }^{99 \mathrm{~m}} \mathrm{Tc}$ and ${ }^{131} \mathrm{I}$ for Single-photon emission computed tomography (SPECT) and ${ }^{90} \mathrm{Y}$ for radiotherapy $[8,13,24]$. They validated the in vivo CLI coupled with OI, PET or SPECT as multimodality imaging strategy for animal imaging using ${ }^{18} \mathrm{~F}-\mathrm{FDG}$ (Figure 2), $\mathrm{Na}^{18} \mathrm{~F}, \mathrm{Na}^{131} \mathrm{I}$, ${ }^{90} \mathrm{Y}-\mathrm{RGD}-\mathrm{BBN}$, and other radio-labeled probes. An increase in $\mathrm{CL}$ signal was observed with the accumulation of the radioactive probes in mouse xenograft tumor. These works demonstrated that CLI imaging highly correlate to the PET or SPECT/CT imaging and indicated that CLI could be used to monitor the distribution of radiopharmaceuticals in vivo. As such, the term, CLI, was coined for this new molecular imaging method.

Besides these early in vivo studies of CLI, Cho et al. described the use of CR imaging as a method for quantitatively measurement of ${ }^{18} \mathrm{~F}$ in a microfluidic chip, which is a new method for monitoring radiochemical synthesis [25]. A lens-coupled CCD system was used for quantitatively imaging the CL produced from ${ }^{18} \mathrm{~F}$ in a microfluidic chip. It provided a tool for investigating and developing microfluidics for radiochemical synthesis.

Generally, this new OI modality does not require change of the procedures or instruments of OI and does not need external excitation. Thereafter different groups have studied on the applications of radionuclides labeled probes in CLI and have showed very encouraging results.

\section{CLI of positron emission radionuclides}

Positron emission radionuclides, such as ${ }^{18} \mathrm{~F},{ }^{15} \mathrm{O},{ }^{13} \mathrm{~N}$, and ${ }^{11} \mathrm{C}$ labeled tracers, have been widely used for diagnosing diseases. As mentioned, the energy of most commonly used PET radionuclides are higher than the threshold energy $(261 \mathrm{keV})$ to produce CL. Therefore, these radionuclides, including the most widely used ${ }^{18} \mathrm{~F}$ and other PET radionuclides, such as ${ }^{13} \mathrm{~N},{ }^{64} \mathrm{Cu},{ }^{89} \mathrm{Zr}$, ${ }^{124} \mathrm{I}$ [26], ${ }^{68} \mathrm{Ga}$ [8], and ${ }^{74} \mathrm{As}$ [27] were thus studied for CLI in different applications.

${ }^{18} \mathrm{~F}-\mathrm{FDG}$ is the most accessible PET probe in clinic and has been widely used for the assessment of tumor glucose metabolism [28]. Now, performed with CLI technology, it could be detected easily by OI $[13,24,29]$. The biodistribution of ${ }^{18} \mathrm{~F}-\mathrm{FDG}$ was dynamically monitored by CLI and PET/CT over the course of time. The ${ }^{18}$ F-FDG accumulations in the brain, tumor, and kidneys observed with CLI was in agreement with the PET images. The optical signal measured by CCD increased proportionally to the radioactivity of ${ }^{18} \mathrm{~F}$-FDG and consistent with the standardized uptake value (SUV) and the percentage of injected dose per gram (ID\%/g) determined from PET at each time point. But interestingly, the kidney/tumor ratio measured by CLI was lower than that obtained by nuclear medicine imaging, likely due to the limited tissue penetration ability of CL, suggesting CLI has limitations for deep tissue imaging and quantification but has good prospect for subcutaneous tumor imaging studies with low interferer of other organ signal. It was also studied that the optical signal of ${ }^{13} \mathrm{~N}$ labeled probes with higher positron energy was higher than the signal of ${ }^{18} \mathrm{~F}$ labeled probes. Consistent with the CR theory, it also showed that the optical signal increases as the energy and refractive index increases.

Besides using ${ }^{18} \mathrm{~F}$-FDG-CLI for tumor imaging, recently, ${ }^{18} \mathrm{~F}$ FDG was used for the in vivo imaging of brown adipose tissue (BAT), which is the tissue for thermogenesis and plays important roles in metabolism and energy expenditure under both healthy and disease status [30]. As such, high CLI quality was obtained when ${ }^{18}$ F-FDG $(200-300 \mu \mathrm{Ci}$ with i.v. injection) was used. It demonstrated that CLI was ideal for BAT imaging study, because the location of BAT is often shallow and situated away from large organs, such as liver, heart, and stomach.

Except for ${ }^{18} \mathrm{~F}-\mathrm{FDG}$, other ${ }^{18} \mathrm{~F}$ labeled probes also could be studied by CLI. Such as ${ }^{18}$ F-FHBG (9-(4- ${ }^{18}$ F-fluoro-3[hydroxymethyl]butyl)guanine) for imaging the reporter geneherpes simplex virus type-1 thymidine kinase (HSV1-tk) [31], and $2-{ }^{18} \mathrm{~F}$-fluoro-CP-118,954 $\left({ }^{18} \mathrm{~F}\right.$ labeled acetylcholinesterase inhibitor for evaluating the reduction in AChE activity which is very important to the patient with Alzheimer's disease (AD) [32].

Some other positron emission radionuclides such as ${ }^{124} \mathrm{I}$ and ${ }^{89} \mathrm{Zr}$ were also studied with CLI by some groups. ${ }^{124} \mathrm{I}$ is a commonly used PET imaging radionuclide for thyroid imaging and ${ }^{89} \mathrm{Zr}$ is a long-lived $\left(t_{1 / 2}: 78.4 \mathrm{~h}\right)$ positron-emitting radionuclide that is generally used for the labeling of monoclonal antibodies (mAbs) for PET imaging. Jeong et al. demonstrated CLI for thyroid gland imaging using ${ }^{124} \mathrm{I}$ [33]. The in vitro cell studies and in vivo imaging both indicated that the CLI of radioactive iodine was good correlated with PET imaging. To assess the potential application of ${ }^{89} \mathrm{Zr}$ CLI in vivo, Ruggiero et al. used the ${ }^{89} \mathrm{Zr}$-labeled $\mathrm{mAb}{ }^{89} \mathrm{Zr}$-desferrioxamine B $\left({ }^{89} \mathrm{Zr}\right.$ DFO-J591) to detect the prostate-specific membrane antigen (PSMA) expression of prostate cancer [26] and subsequently reported another ${ }^{89} \mathrm{Zr}$ labeled $\mathrm{mAb},{ }^{89} \mathrm{Zr}$-DFO-trastuzumab, for target-specific and quantitative CLI imaging of HER2/neupositive tumors in vivo [34]. Recently, ${ }^{89} \mathrm{Zr}$ CLI was also used for quantitative non-Hodgkin's lymphoma (NHL) imaging 


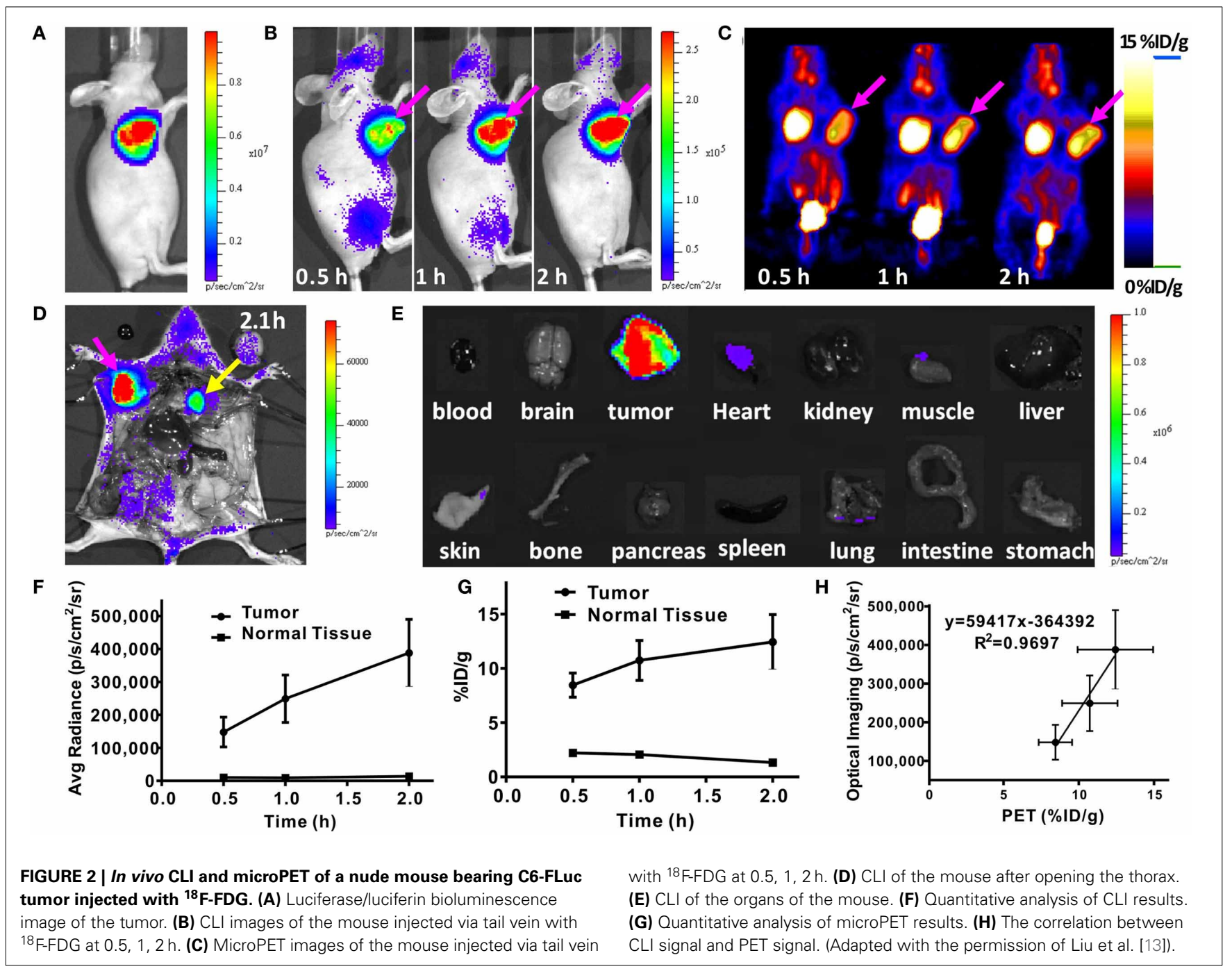

using ${ }^{89} \mathrm{Zr}$-desferrioxamine-rituximab in humanized huCD20 transgenic mouse models [35].

Finally, all of these studies concluded that the measured CL signal of these positron emission radionuclides showed a positive correlation with the radioactivity of the radionuclides, moreover, showed a good correlation between CL signal and PET signal in vivo. This suggesting that CLI is a potentially quantitative and sensitive imaging method as PET for biological research, such as metabolism imaging, tumor-target imaging, report gene imaging and antibody imaging.

\section{CLI of beta emission radionuclides}

CR has been applied for countering $\beta^{-}$emitter since such radionuclides cannot be detected directly because of the low penetration of the $\beta^{-}$particles. During the development of drugs for targeted radiotherapy, $\beta^{-}$emitting radionuclides were shown to have great therapeutic benefit due to the limited path length and high linear energy transfer (LET) of the $\beta^{-}$particles. Such radiopharmaceuticals were promising for cancer therapy [36]. However, the biodistribution and uptake of the pure $\beta^{-}$emitterlabeled radiopharmaceuticals cannot be imaged directly using conventional SPECT or PET. Thus, CLI has a great advantage for imaging such $\beta^{-}$radionuclides.

${ }^{131} \mathrm{I}$ is a commonly used $\beta^{-}$and $\gamma$ emission radionuclide and can be used for therapy and imaging of thyroid disease since iodine ion accumulates in the thyroid [37].

${ }^{131} \mathrm{I}$ is a good theranostic isotope, but the SPECT imaging of it usually shows low resolution since the $\gamma$-ray energy of ${ }^{131} \mathrm{I}$ is too high $(364 \mathrm{KeV})$ for SEPCT imaging. However, combined high-resolution OI technique, CLI shows good prospect of ${ }^{131} \mathrm{I}$ imaging with high resolution. In another study, CLI was used for visualizing the expression of let-7 in lung adenocarcinoma A549 cells transfected with human sodium/iodine symporter-ras gene (hNIS-RU) [38]. Then, 3D CLI tomography was demonstrated to quantify tumor-associated aminopeptidase $N$ (APN/CD13) expression with ${ }^{131}$ I labeled Asn-Gly-Arg peptide ( ${ }^{131} \mathrm{I}-\mathrm{NGR}$ ) [39]. All of the results showed that CLI could non-invasively detect miRNA and aminopeptidase $N$ expression and consistent to the SPECT imaging data. Liu et al. used $\mathrm{Na}^{131}$ I for thyroid CLI, which showed the accumulation of the ${ }^{131} \mathrm{I}$ in thyroid as well as showed in SPECT/CT [13]. After the in vivo CLI and SPECT/CT, the mice were sacrificed to expose the thyroid and other organs. 
Further CLI revealed that the thyroid could be clearly differentiated with high resolution. This suggests that the CLI imaging could be used for surgery guidance, which will be discussed in section CLI Guided Surgical Resection.

${ }^{90} \mathrm{Y}$ and ${ }^{32} \mathrm{P}$ both are pure $\beta^{-}$radionuclides with long halflife $\left(64 \mathrm{~h}\right.$ for ${ }^{90} \mathrm{Y}, 14.29$ days for ${ }^{32} \mathrm{P}$ ) and high $\beta^{-}$particles energy. They were widely used for radiotherapeutic or diagnostic purposes. However, because they lack gamma photons, only Bremsstrahlung radiation can be used in SPECT imaging for them, but the resolution of such Bremsstrahlung SPECT imaging is too low to be used. As such, CLI provides new strategy for such pure $\beta^{-}$radionuclide imaging. The CLI was successfully used for dynamically monitoring the distribution and clearance properties of ${ }^{90} \mathrm{Y}$ labeled probes in tumor bearing mice $[13,40]$, metabolism of ${ }^{32} \mathrm{P}$-phosphate in plant [41], and the spatial and temporal distribution of ${ }^{32} \mathrm{P}$ labeled Adenosine triphosphate ( ${ }^{32} \mathrm{P}$-ATP) uptake in vivo [42].

Recently, Wang et al. reported that ${ }^{198} \mathrm{Au}$ has the CR effect. A novel ${ }^{198} \mathrm{Au}$-Gold Nanocages (AuNCs) were synthesized for CLI. This radioactive nanoparticles were shown to accumulate well in the tumor and emitted luminescence with continuous wavelengths in the visible and near-infrared (NIR) regions for CLI [43].

In a summery, the CLI could be used for most kind of $\beta^{-}$radionuclides especially for pure $\beta^{-}$radionuclides imaging in vivo. It shows great advantages of high resolution to Bremsstrahlung SPECT imaging or high energy $\gamma$ camera imaging. This is very helpful for radionuclide therapy research with high resolution and sensitivity.

\section{CLI of alpha emission radionuclides}

Recently, an alpha emission radionuclide, ${ }^{225} \mathrm{Ac}$, was studied for CLI by Ruggiero et al. and was found to give very high intense optical signal [26]. ${ }^{225}$ Ac releases $\alpha$-particles with high energies in the range of 5,021 to $5,830 \mathrm{keV}$. However, the $\alpha$ particles are known to travel at velocities below the threshold for CR, although the luminescence signal could be observed by an OI system. The possible explanation was that the optical emissions originate from a series of short-lived, $\beta^{-}$emission daughter nuclides, including ${ }^{213} \mathrm{Bi},{ }^{209} \mathrm{~Pb}$ and ${ }^{209} \mathrm{Tl}$. Then, by using the simulation toolkit Geant4, the CL output from the decay chain of ${ }^{225} \mathrm{Ac},{ }^{230} \mathrm{U},{ }^{213} \mathrm{Bi}$, ${ }^{212} \mathrm{Bi}$, and ${ }^{212} \mathrm{At}$ were studied. As a result, ${ }^{230} \mathrm{U}$ and ${ }^{211}$ At produce little CL because of more than 10 -years half-lives. ${ }^{213} \mathrm{Bi}$ and ${ }^{212} \mathrm{Bi}$ produce an order of magnitude more $\mathrm{CL}$ than ${ }^{18} \mathrm{~F}$, but there are many more than $1 \mathrm{MeV}$ high-energy $\gamma$-rays in the decay chains that also produce CL. The CL produced by these high-energy $\gamma$-rays depends on the volume of medium thus not images the location of ${ }^{213} \mathrm{Bi}$ and ${ }^{212} \mathrm{Bi}$. For ${ }^{225} \mathrm{Ac}$, it can be imaged by CLI since there are $\beta^{-}$radionuclides in its decay chain. However, these daughter radionuclides may no longer attach to the target and thus the CL may be delayed from the initial ${ }^{225}$ Ac decay, possibly decreasing the correlation of the ${ }^{225}$ Ac labeled drug location and light source [12].

\section{CANCER THERAPY MONITORING}

Because PET and SPECT have been approved and used for cancer therapy monitoring, and the same radionuclide labeled probe used for these imaging modalities may also be used for CLI. $\mathrm{Xu}$ et al. demonstrated a proof-of-concept study of monitoring cancer drug therapy with CLI [44]. They used two common radiotracers, $3^{\prime}-\left[{ }^{18} \mathrm{~F}\right]$ fluoro- $3^{\prime}$-deoxythymidine $\left({ }^{18} \mathrm{~F}\right.$-FLT $)$ and ${ }^{18} \mathrm{~F}-\mathrm{FDG}$, to perform CLI and PET in therapy monitoring. Two murine tumor models, a lung cancer cell line H460 and prostate cancer cell line PC3 were treated with bevacizumab vs. vehicles. Subsequently, they selected Bevacizumab as a therapeutic agent to treat mice bearing $\mathrm{H} 460$ xenografts, and then they performed CLI and PET to monitor the therapy effect with ${ }^{18} \mathrm{~F}$ FLT. Excellent correlations were found between CLI and PET for the monitoring of cancer therapy [45]. Robertson et al. used CLI and PET to compare the efficacy of MLN4924, a phase 2 oncology therapeutic [46]. The CLI data correlated well with PET and showed dynamic sensitivity. That means the CLI could be used for tumor therapy monitoring in biology studies. This will give big benefit for those labs without PET or SPECT imaging instruments.

\section{GUIDED SURGICAL RESECTION}

Because of its unique imaging characteristics, CLI has the potential to be used as a very efficient method for identifying small tumor masses and defining the localization or extent of tumor lesions with accurate delineation of tumor margins. Accordingly, it can potentially be used to guide surgical resection. Holland et al. demonstrated the use of CLI as a tool to guide the surgical resection of tumors [34]. A series of CLIs was recorded before, during, and after surgical resection of the BT-474 (HER2/neu positive) tumor after $144 \mathrm{~h}$ of ${ }^{89} \mathrm{Zr}$-DFO-trastuzumab injections. The optical signal intensity of the image recorded after surgical incision and exposure of the tumor increased markedly. After surgery, the complete loss of optical signal emitted from the mouse was observed at the former location of the tumor. Overall, these pre-, post-, and intraoperative optical images showed the potential for using CLI as a surgical resection guide.

Thorek et al. investigated CLI for dynamic mapping and guiding surgical resection of lymph nodes (LNs) in animal models using intradermal administration of ${ }^{18} \mathrm{~F}-\mathrm{FDG}$ [47]. The transit and accumulation of the ${ }^{18} \mathrm{~F}-\mathrm{FDG}$ at the sacral nodes provided sufficient CR to guide surgical intervention and resection of LNs. The intradermal injection isolated the ${ }^{18} \mathrm{~F}-\mathrm{FDG}$ to the draining lymphatic channels and their associated LNs. The sacral node could be seen after removal of the skin (i.e., in an intraoperative setting), before any of them was revealed by surgical incision.

Another very important tool for surgery guidance is the use the endoscopy, which is reviewed in the next section.

\section{ENDOSCOPIC IMAGING}

Endoscopy has played a significant role in the diagnosis of gastrointestinal diseases and surgery guidance. However, because traditional endoscopic imaging is anatomic imaging, it is sometimes difficult to distinguish between some diseases with similar anatomical abnormalities or the boundaries between tumor and normal tissue. Multimodality imaging combining anatomical and functional imaging methods has been a very important tool for cancer diagnostic and surgery guidance. Thus, endoscopy is often 
combined with anatomical and functional imaging to achieve a comprehensive interpretation and understanding [48].

Our group combined different optical fibers and endoscopy with CCD to investigate whether CL can be detected with optical fibers or endoscopes [49]. We reported that the throughputs of the optical fibers were different depending on the core diameters of the optical fibers. The throughput of the optical fiber with $6 \mathrm{~mm}$ core diameters is relatively higher compared to others used in the study. The limiting concentration of ${ }^{18} \mathrm{~F}-\mathrm{FDG}$ that could be reliably detected with CL was as low as $1 \mu \mathrm{Ci}$. This allowed fiber optics to be used for detecting the CL from the organ of interest in the body. This optical fiber imaging system was then used to detect the CL signal from different parts of C6 glioma tumor bearing mice after intravenous injection of ${ }^{18}$ F-FDG. Consistent with PET, optical fiber imaging found relatively high signals from the subcutaneous tumor and brain, indicating a high uptake of ${ }^{18} \mathrm{~F}-\mathrm{FDG}$ in these tissues. However, the image obtained from this system only displayed as a facula because the CCD was separate from the ocular of the optical fiber. We then improved this system and investigated the proof-of-concept of using Cerenkov luminescence endoscopy (CLE) to guide cancer surgical resection [50]. The fiber-based CLE system comprised an imaging optical fiber coupled to a highly sensitive intensified CCD camera and showed high spatial resolution and sensitivity. This CLE system was used to demonstrate surgical resection guidance in a xenograft mouse model bearing C6 glioma tumors. The study successfully provides additional support for the use of CLI as a promising modality for endoscopic molecular imaging.

\section{EXTERNAL RADIOTHERAPY APPLICATIONS}

A number of recent of studies have demonstrated the feasibility of collecting CL that is excited by an external radiation beam. As mentioned previously, the optical signal intensity increases as the energy of the charged particles increases. The commonly used linear accelerator works with high energy of more than $1 \mathrm{MeV}$, which is far greater than the threshold energy in water of $261 \mathrm{keV}$. Thus, the liner electron beam might produce a stronger CL. In some external radiotherapy beam dosimetry instruments, such as plastic scintillators, the CL has sometimes been considered as an unwanted background signal [51-55]. However, CL produced by charged particles, and monitoring the CL generated in the medium can potentially be used for dosimetry measurement. Jang et al. fabricated a fiber-optic CR sensor (FOCRS) for measuring the CR produced by a therapeutic proton beam. The CL measured increased linearly with the increasing dose rate of the proton beam [56]. They further fabricated a plastic optical fiber for the same purpose [57]. When a photon beam passes through a medium, it reacts with the medium and produces Compton electrons, which can travel at a speed higher than light in this medium and thus produce CL. The depth dose distribution of the photon beam depends on the electron fluxes at each depth of a water phantom because Compton scattering is the predominant interaction for the photons. Additionally, according to the theory of the Cerenkov effect and relativity, the intensity of CR is directly proportional to the depth doses for a therapeutic photon beam. Finally, the percentage depth doses (PDD) of the photon beam were obtained using the CR generated in plastic optical fiber over a depth of $8 \mathrm{~mm}$, with only near-surface region correction.

Glaser et al. investigated the use of projection imaging for external radiotherapy photon beam profiling [58]. By using Monte Carlo simulations for correction, the CLI based beam profiles were compared to PDD, and the reference dose distribution was generated from the radiotherapy treatment planning system (TPS). As a result, the optical signal intensity of CLI was directly correlated to the deposited dose and demonstrated the CLI to profile $\mathrm{x}$-ray photon external radiotherapy beams in water. However, the $2 \mathrm{D}$ projections of the CLI appeared blurred and yielded values higher than expected just outside the beam edge and lower than expected values just inside the beam edge. This group also investigated the full 3D CLI beam profiling and quality assurance (QA) for x-ray photon beams. They explored an optical-based dose imaging approach that allows $2 \mathrm{D}$ projection imaging in a fast timeframe and created full 3D profiles after tomographic reconstruction from multiple projections. This approach reinforced the importance on patient-specific treatment plans during external radiotherapy.

Similar to $\beta^{-}$particles, the high energy electron beam which was commonly used for external radiotherapy, could also produce $\mathrm{CL}$ according to the theory of CR. Axelsson et al. systematically studied the CL generation by an external electron beam with different energies using a water-based phantom [59]. The intensity of CL produced by an external electron beam increased along with increasing the electron energy. The spectrum study also demonstrated that the spectrum of CL generated by external electron beams is similar to the CL spectrum produced by radionuclides but contains blue-shifted light. Furthermore, the blue-dominated spectral of CL can excite a fluorophore called protoporphyrin IX (PpIX) which selectively accumulates in brain tumors and has photosensitizing properties that can be used for photodynamic therapy (PDT). Thus, external electron beams could be used to achieve PDT due to Cerenkov effect.

Because the oxygenation of the tumor tissue plays an important role during radiation therapy [60], Axelsson et al. measured the hemoglobin oxygen saturation $\left(\mathrm{StO}_{2}\right)$ non-invasively in tumor tissue in vivo [61]. The total hemoglobin concentration and hemoglobin oxygen saturation could be estimated by measuring the absorption and fitting the spectrum of the CL. This methodology enables the quantitative analysis of hemoglobin concentration and oxygen saturation and provides real-time physiological information about the tissue during external radiation therapy. Based on this work, they further demonstrated the non-invasive and quantitative verification of the tissue $\mathrm{StO}_{2}$ and oxygen partial pressure $\left(\mathrm{pO}_{2}\right)$ in vivo. The tomographic imaging of $\mathrm{StO}_{2}$ and oxygen partial pressure $\left(\mathrm{pO}_{2}\right)$ in deep tissues was studied using Monte Carlo analysis and tissue oxygenation phantoms, respectively. The emission lifetime of some oxygen-sensitive phosphorescence was directly related to the tissue $\mathrm{pO}_{2}$. The $\mathrm{CL}$ induced by the external radiotherapy beam could excite these probes and thus be used to measure the tissue $\mathrm{pO}_{2}$. This study indicates that the $\mathrm{StO}_{2}$ and $\mathrm{pO}_{2}$ in tumor tissue can be quantitatively measured by detecting the CL during radiation therapy [62]. Following this approach, they investigated the tomographic images of phosphorescence excited by CL using 
a series of phantoms with various oxygenation states [63]. The successful $\mathrm{pO}_{2}$ distribution tomography during external radiotherapy may significantly impact radiotherapy studies, although this technique still needs further optimization for faster and more sensitive acquisition.

Another use of CL in external radiotherapy was radiation dose calibration and quality assessment. This work was reported by Glaser et al. in 2013 [64]. They investigated a high speed and high-resolution full 3D tomography of CL induced by external radiotherapy beams to estimate volumetric dose distributions in a pure water phantom. It is very important to do the dose distribution calibration and quality assessment before external radiation therapy because the accurate dose is needed to achieve the desired therapy effect with minimal side effects. This noninvasive technology was not only able to evaluate the energy deposition of charged particles, but it also could be applied to additional applications utilizing high-energy ionizing radiation.

\section{INTERNAL EXCITATION SOURCE}

Fluorescent imaging has been widely used for biology research. In fluorescence imaging, the fluorophores are excited by an external light, and thus, CL may serve as an internal source for exciting these different fluorophores for fluorescent imaging. Liu et al. demonstrated this concept by using quantum dots (QDs) excited by ${ }^{131}$ I for OI [65]. Three type of CdSe/ZnS core/shell QDs (QD655, QD705, and QD800) were selected and mixed with $\mathrm{Na}^{131} \mathrm{I}$ for in vitro and in vivo OI studies. As a result, three fluorescence emission peaks at $650 \mathrm{~nm}, 705 \mathrm{~nm}$ and $800 \mathrm{~nm}$ were observed separately, corresponding to the characteristic emissions of these QDs. For the in vivo imaging studies, $\mathrm{Na}^{131} \mathrm{I}$ was mixed with QD655, QD705, and QD800, and the resulting mixture was injected subcutaneously into different locations of the mice. Images were then obtained using different filters for each QD's emission and were displayed with different colors. Thus it gives a multi-colored imaging in one time for different drugs. Since the spectrum of CR is very wide, it not only excites the QDs but also could excite much more fluorophores. For example, $\mathrm{CL}$ internal excites radioluminescent nanophosphors (RLNPs, $\mathrm{Ba}_{0.55} \mathrm{Y}_{0.3} \mathrm{~F}_{2}: \mathrm{Tb}^{3+}$, and $\mathrm{Ba}_{0.55} \mathrm{Y}_{0.3} \mathrm{~F}_{2}: \mathrm{Eu}^{3+}$ ) for animal fluorescence imaging [66]. This provided a novel CL excited fluorescence OI method.

In another interesting study, Ran et al. demonstrated CL utility for internal excitation and the photoactivation of luciferin 1-(4,5dimethoxy-2-nitrophenyl) ethyl ester (DMNP-luciferin) in vivo [67]. In this study, ${ }^{18} \mathrm{~F}-\mathrm{FDG}(0.4 \mathrm{mCi})$ was injected into the mice bearing luciferase-expressing breast tumors before DMNPluciferin administration to allow the ${ }^{18} \mathrm{~F}-\mathrm{FDG}$ to distribute to the tumor properly. Optical images were obtained to evaluate the CL excitation effect after injecting the DMNP-luciferin. Their results indicate that the CL from ${ }^{18} \mathrm{~F}-\mathrm{FDG}$ activates the DMNP-luciferin to release the luciferin for bioluminescence imaging at significantly higher levels even than that of with $365 \mathrm{~nm}$ UV activation. This work shows the successful application of CL as an internal excitation for photoactivation.

By using Cerenkov energy transfer theory, a targeted and activatable fluorescent probe, which could be excited by CL, was established to detect and quantify the activities of some molecules, like enzyme [68]. This strategy was named secondary Cerenkov-induced fluorescence imaging (SCIFI). In detail, the gold nanoparticles (AuNPs) as a quencher were conjugated to a fluorescein (FAM) with a biocompatible activatable peptide linker. The linked peptide could be cleaved by matrix metallopeptidase-2 (MMP-2) enzyme in vivo. After this activatable probe was injected, ${ }^{18}$ F-FDG was injected to excite the FAM. Once the linked peptide was cleaved by MMP-2, the AuNPs will not quench the FAM and the specific emission of FAM could be detected and imaged with OI instrument. Thus the activity of MMP-2 enzyme was detected according to the SCIFI emission peak. This strategy lends CLI for further investigation of target-specific and cleavable multiple probes in vivo imaging.

Recently, Kotagiri et al. demonstrated that using DNA as a spacer to control the distance between QD655 and ${ }^{64} \mathrm{Cu}$ produces the CL to excite the QDs [69]. To test the distance-dependent $\mathrm{CL}$ energy transfer, QD655 and ${ }^{64} \mathrm{Cu}$ distance was set between $1.15 \mathrm{~nm}$ to $31.15 \mathrm{~nm}$ using DNA as a linker. The luminescence emission increased as the distance between QD655 and ${ }^{64} \mathrm{Cu}$ increased from $1 \mathrm{~nm}$ to $31 \mathrm{~nm}$. Additionally, $\mathrm{Cu}^{2+}$ has a significant quenching efficiency on QD luminescence, and thus can be used both as a CLI probe and a luminescence quencher.

The ultraviolet (UV) light is usually used to excite the fluorophores or activate the chemical reaction in some OI methods. But it is limited by insufficient penetration of UV light in tissue when it is used for in vivo imaging, and this imaging cannot be effectively used in deep tissues. Thus, CL was considered as a good internal excitation alternative to UV. In a word, the CLI has great potential applications as an internal excitation source in vivo to activate the chemical reactions, QDs, PDT drugs or other fluorophores for different purpose.

\section{HUMAN APPLICATION}

The first human use of CLI was demonstrated for human thyroid imaging by Spinelli et al. [70]. They obtained the CLI images for a patient with hyperthyroidism that was treated with $15 \mathrm{mCi}$ of ${ }^{131}$ I. The CLI was acquired using an electron multiplied CCD (EMCCD) OI system in a light tight dark room without windows and neon lamps. The patient and the room door were covered by thick black curtains to avoid any possible external light. After treatment with ${ }^{131} \mathrm{I}$ for $24 \mathrm{~h}$, the patient was imaged for $2 \mathrm{~min}$, with the camera focusing on the head and neck region of the patient. A significant CL signal was observed at the thyroid region and was confirmed by a nuclear medicine physician with extensive experience in thyroid imaging (Figure 3). This first human CLI demonstrated that it is possible to obtain a planar image from the superficial organs of patients with radioactive probes. This method was named Cerenkography. Recently, another human superficial organ CLI was reported to successfully detect the diagnostic doses ${ }^{18}$ F-FDG accumulations in axillary LNs using sensitive optical equipment [71]. Different with the thyroid CLI, the axillary LNs CLI was performed with a diagnostic dose of radioactive probe by using a more sensitive optical camera. This demonstrated that the CLI technique has significant potential applications by visualizing the optical emissions of radioactive tracers accumulated in human organs or tumors and will be more sensitive and accurate with the development of 


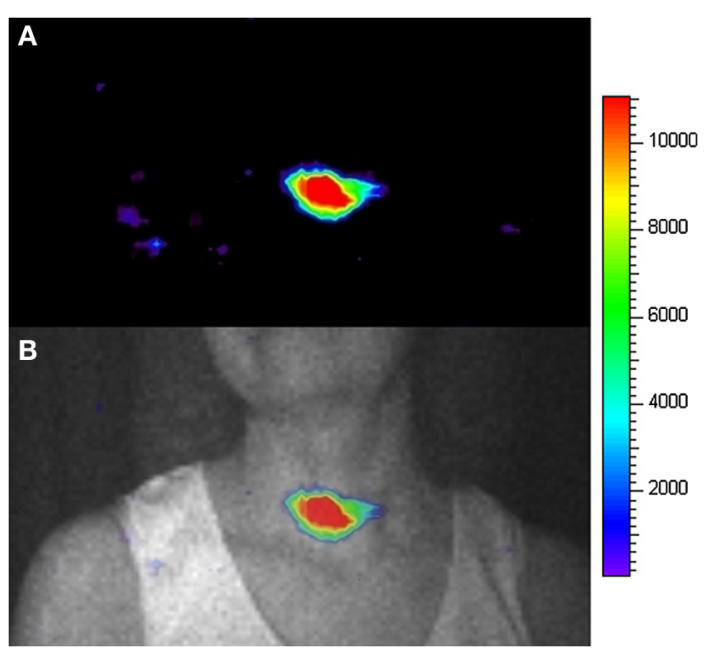

FIGURE 3 | The CLI image (A) and the overlay between the photographic image (gray color) and the CLI image (colorful) (B) of the human thyroid. (Adapted with the permission of Liu et al. [65]).

optical instruments. There should be a further development of CLI in human applications.

\section{OPTIMIZATION OF CLI}

The CL intensity is relatively weak and the most intensive CL occurs in the spectrum of the UV and blue wavebands because of the inverse square wavelength dependence. However, UV and blue light are usually easily scattered and absorbed by biological tissues [72]. Thus, for in vivo imaging, the penetration and scattering of CL will complicate the detection and quantification of CLI. As such, several groups have sought to optimize the CLI by shifting the inherent emission to the NIR wavelengths that have better penetration and lower scattering.

A high Stokes-shift QD nanoparticle, Qtracker705, which can produce highly red-shifted photonic emissions of $705 \mathrm{~nm}$ by excitation at $350 \mathrm{~nm}$, was used for CR energy transfer (CRET) imaging by Doehager et al. [73]. The absorption spectrum of Qtracker705 overlaps the UV/blue emissions of CR, and thus, it absorbs the CL and produces light with a peak centered at $705 \mathrm{~nm}$. They demonstrated that efficient energy transfer could be detected with ${ }^{64} \mathrm{Cu}$ and ${ }^{18} \mathrm{~F}$ mixed with Qtracker705 (Figure 4). The CRET ratio was calculated and normalized to quantify the imaging signals as a quotient of the light detected within a spectral window centered on the Qtracker705 emission divided by the light detected within a spectral window of the CR emission. ${ }^{18}$ F-FDG CLI transferred by Qtracker705 nanoparticles showed high CRET ratios in vitro and in vivo. Additionally, the donor intensity decreased and CRET quasilinear increased with increasing concentrations of Qtracker705 within the narrow concentration range. This report demonstrates that the UV/blue CL can be absorbed and transferred to far-red and NIR emission by the QD nanoparticles. This result suggests that other types of energy transfers could be potentially applied to other isotopes that produce CL in the media.

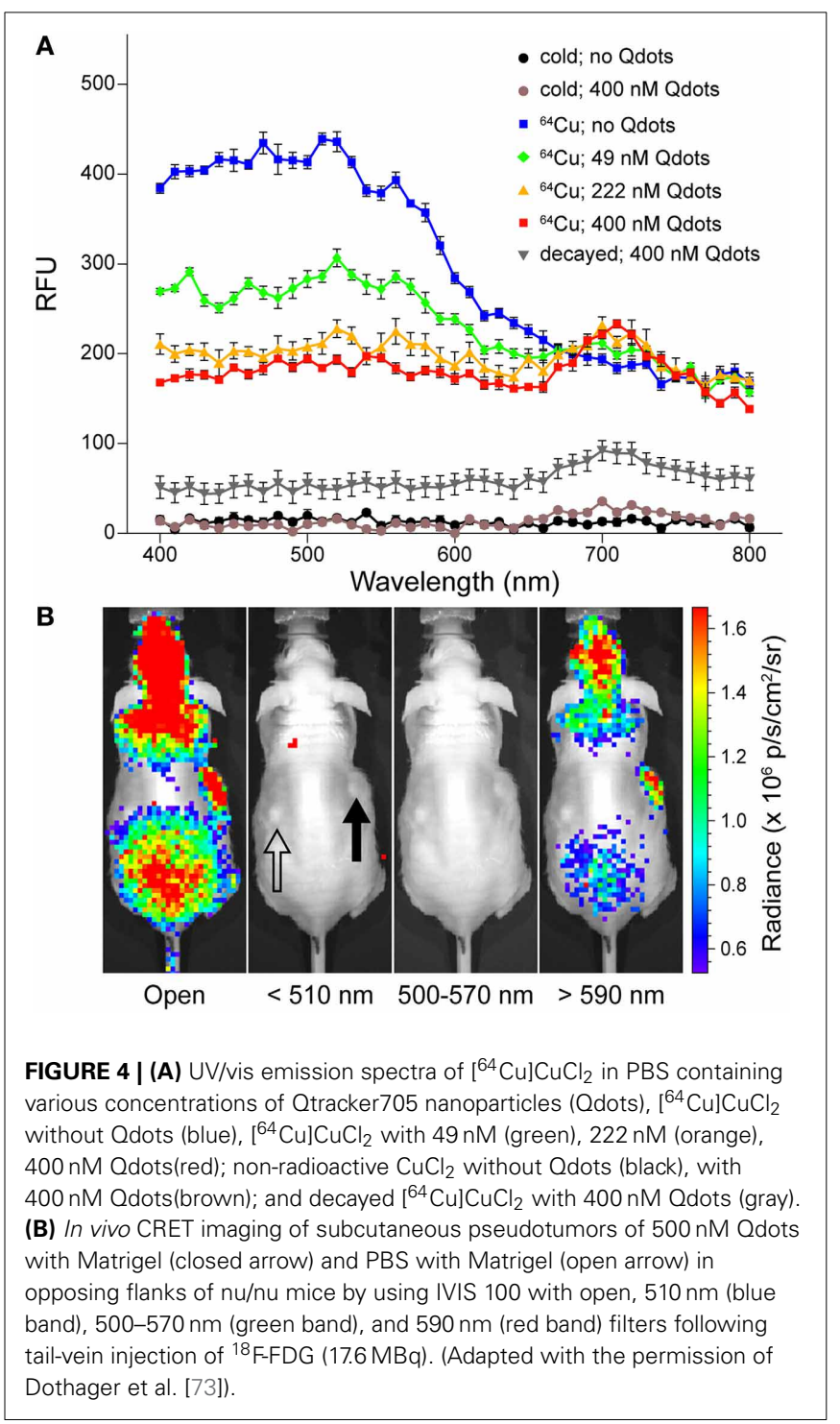

Lewis et al. reported another CL transfer study using fluorophore agents as a converter [27]. In this study, some fluorophores with potential preclinical applications were used, such as europium (III) chloride hexahydrate $\left(\mathrm{EuCl}_{3}\right)$, thulium (III) chloride hexahydrate $\left(\mathrm{TmCl}_{3}\right)$, gadolinium (III) chloride hexahydrate $\left(\mathrm{GdCl}_{3}\right)$, indocyanine Green (ICG), and gadopentetate dimeglumine. Each of these fluorophores was mixed with $14 \mu \mathrm{Ci}$ of ${ }^{18} \mathrm{~F}$ or ${ }^{74}$ As per well in a black plastic well plate, and the spectrum was tested. The luminescence of the wells with the fluorophores and ${ }^{18} \mathrm{~F}$-FDG were stronger than the well with ${ }^{18} \mathrm{~F}$-FDG alone. The luminescence peaks of europium (III) chloride hexahydrate excited by ${ }^{18} \mathrm{~F}$ and ${ }^{74}$ As were near $600 \mathrm{~nm}$, and $700 \mathrm{~nm}$, respectively, which have stronger penetration in tissue. Additionally, the luminescence peak near $600 \mathrm{~nm}$ of $\mathrm{EuCl}_{3}$ appeared to be red-shifted with ${ }^{74}$ As excitation compared to ${ }^{18} \mathrm{~F}$. A weak luminescence was also observed in a well containing only fluorophores and surrounded by wells containing ${ }^{74}$ As. This luminescence may attribute to the excitation by lower energy photons from ${ }^{74}$ As. 
This phenomenon observed may have potential applications in the future.

To optimize the CLI, Spinelli et al. investigated the theoretical enhancement of photon intensity in the NIR wavelength band based on a photon propagation diffusion model [74]. Their results showed that despite the low intensity of CL in the NIR band, the luminescence transferred from the tissues was stronger than that in the visible band. According to this, the CCD that was optimized for the NIR range detection would yield a higher optical signal and thus enhance the imaging quality of CLI. By using this optimized CCD, the enhancement was approximately $35 \%$ and was more significant when the source was located in deeper tissue. Overall, by using energy transfer strategy, the UV/visible band of CL could be absorbed by some fluorophores and could emit NIR light, which could be detected by optimized CCDs more effectively. However, the absorber could be further developed for improved efficiency and a greater red shift.

In summary, the CLI quality could be significantly improved by using some red shift fluorophores, but the energy transfer efficiency still not high enough that causing the insufficient emission at NIR window. And even the CL energy was transferred to NIR emission, the tissue penetration of the light still limit by scatter and absorption and thus still cannot be used in deep organ imaging. Another shortage of this optimization strategy is the use of fluorophores, which still have not been approve for clinical use while most of the CLI probes have been used in clinical for a long time. Once conjugated with fluorophores, the CLI probes cannot be used in clinical study anymore. Thus, these optimize strategy still not very ideal enough and need further optimization.

\section{TOMOGRAPHY}

As an imaging method, two-dimensional (2D) planar imaging lacks depth information, while three-dimensional (3D) optical tomography provides more precise spatial distribution and information about the probes and diseases. Therefore, some studies described the use of 3D tomography reconstruction for CLI and named the technique CLT. Li et al. first proposed that CLT could be used to evaluate the distribution of radioactive tracers in vivo by surface measurement of CL [75]. The CLT was reconstructed by using an inverse algorithm and the diffusion equation (DE) adapted from that used for bioluminescence optical tomography (BOT) [76]. The phantom or animal studies with radioactive probe were placed between two mirrors inside a very sensitive OI system. The CCD camera simultaneously captured images of the $695-770 \mathrm{~nm}$ narrowband CL from the top and two side surfaces of the phantom or animal using the mirrors. Then, the images was reconstructed quantitatively by $3 \mathrm{D}$ tomography and compared with PET imaging. The distribution of ${ }^{18}$ F-FDG in the animal reconstructed by CLT was concordant with that obtained by PET. However, the spatial resolution of CLT is much lower than PET because of light scattering. In this method, the number of measurement, photon propagation and the spectral information all affect the image quality of the CLT. A narrower bandwidth filter, multispectral measurements and higher sensitivity CCD help to improve image quality in future developments of the technique.

Another reconstruction approach, multispectral diffuse CLT (msCLT), was investigated by Spinelli et al. [77]. The msCLT was reconstructed by a set of planar images acquired using a series of narrowband (FWHM: $20 \mathrm{~nm}$ ) emission filters from 560 to $660 \mathrm{~nm}$. All of these planar images were reconstructed to 3D images based on the diffusion approximation (DA) theory. By using chicken breast and mouse muscle simulations, the resolution was estimated with different depths of light sources by msCLT and showed that the resolution was approximately $1.5 \mathrm{~mm}$ at $6 \mathrm{~mm}$ depth and decreased with increasing depth. The 3D biodistribution of ${ }^{32} \mathrm{P}$-ATP was obtained using msCLT and was concordant with that obtained in MRI images. As this work demonstrates, msCLT could improve the reconstruction quality, but the imaging time was relatively longer because a series of multispectral images must be obtained using a set of filters.

In contrast to homogeneous and multispectral-based methods, $\mathrm{Hu}$ et al. performed in vivo 3D-CLT using a heterogeneous mouse model with an implanted $\mathrm{Na}^{131}$ I radioactive source consisting of a glass tube in the mouse abdomen [78]. The images were obtained with a 695-770 nm narrowband emission filter and were reconstructed by the hp-finite element method (hp-FEM) based on DE. They quantitatively reconstructed the localization and intensity of the radioactive source with a gradient concentration of $\mathrm{Na}^{131}$ I. The reconstructed CLT images were merged with the micro-CT image for multimodality imaging to obtain more information. The precision of the position reconstructed by $3 \mathrm{D}$ CLT was compared with the position on the micro-CT and SPECT images. The result showed that the reconstructed CL intensity correlated with the radioactivity and the SPECT signal of ${ }^{131}$ I. Additionally, this $3 \mathrm{D}$ reconstruction method is more accurate than the homogeneous method. For further CLT study, they improved the CLT/CT reconstruction method and used it to image the ${ }^{131}$ I uptake in mouse thyroid glands (Figure 5) [79]. They investigated a more efficient, semi-quantitative hybrid spectral CLT because it does not need a filter. The results of both the implantation mouse model and the ${ }^{131} \mathrm{I}$ uptake in mouse thyroid were encouraging, with reduced acquisition and image reconstruction time. Furthermore, the reconstructed position and quantification of the ${ }^{131}$ I source in the phantom was more accurate than single-spectral CLT but a little less accurate than msCLT. This research group then presented a SPECT-guided reconstruction method for CLT, in which prior information of the permissible source region from the SPECT imaging results was incorporated to reduce the inverse reconstruction problem [80]. The experimental results showed that the reconstruction quality and spatial resolution were improved.

Zhong et al. investigated another CLT/CT reconstruction with third-order simplified spherical harmonic (SP3) approximations and whole-body tomography imaging $[81,82]$. They established the large-scale linear equations for CLT via the SP3 method and demonstrated better imaging quality and reconstruction efficiency than those from DA in biological tissues. Whole-body CLT of small animals was successfully performed. For faster reconstruction speed, they investigated another CLT method with geometric row scaling and $\mathrm{L}_{1 / 2}$ regulation [83].

In summary, all of these multi-view or multi-spectral CLT methods provide $3 \mathrm{D}$ distribution of the radioactive probe in vivo and correlated well with PET or SPECT images. Further work is needed to improve sensitivity and accuracy because CL is very 


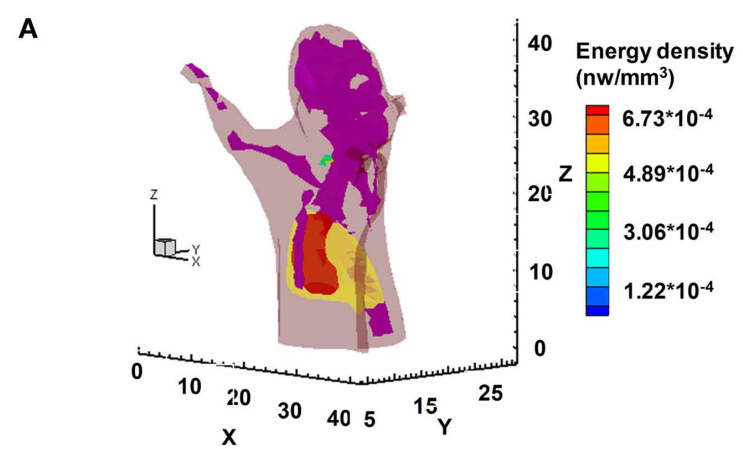

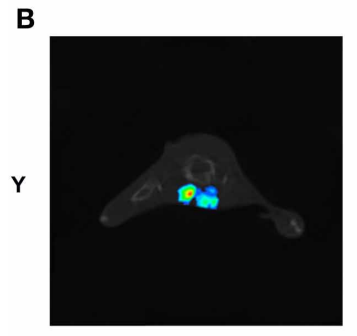

$\mathbf{X}$

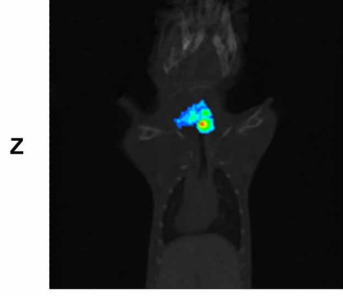

$\mathbf{x}$

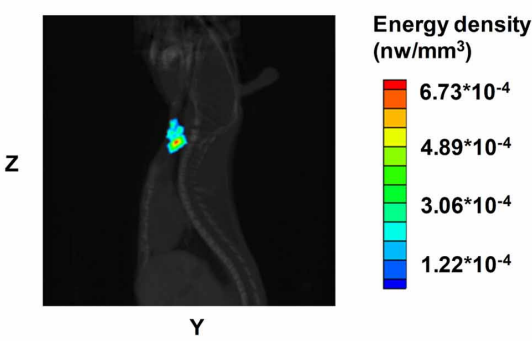

FIGURE 5 | The CLT images of athymic nude mice with tail vein injection of $450 \mu \mathbf{C i} \mathbf{N a}^{131}$ I. (A) The 3D rendering of the $\mathrm{Na}^{131}$ I uptake in the mouse thyroid. (B) The CLT reconstruction results of hybrid spectral CLT of $\mathrm{Na}^{131}$ I uptake in the mouse thyroid in horizontal, coronal, and sagittal views respectively. (Adapted with the permission of $\mathrm{Hu}$ et al. [79]). weak and its intensity occurs mostly in the short wavelength band, which is easily scattered or absorbed by tissue.

\section{LIMITATIONS OF CR}

Given by the CL theory and the experiments results, the widespread implementation of CL in various applications is still challenging. The first issue of CL is the relatively weak optical signal intensity, which could result in difficulties in high sensitivity detection or imaging. As we described in the second section of this review, the signal is approximately 2.4 photons per decay for ${ }^{18} \mathrm{~F}$. Accordingly, if $100 \mu \mathrm{Ci}$ of ${ }^{18} \mathrm{~F}$-labeled probe is injected for an imaging study, the total optical flux is only approximately $9 \times 10^{6}$ photons/s. The second limitation of CL is insufficient penetration. The CL spectrum is continuous and ranges from the UV to NIR wavelength, and its intensity is related to wavelength by $1 / \lambda^{2}$. Thus, the most intensive CL is in the spectrum of the $\mathrm{UV} /$ blue band, which is easily scattered and absorbed by biological tissues. The NIR emission of CL is quite weak, though it has higher capability to penetrate biological tissues. Therefore, only a small portion of CL emitted from the radionuclide in vivo can be detected by OI systems. Thirdly, most of the absorbance of light by tissues is caused by hemoglobin in the blood. Therefore, the organs containing more blood absorb a significant amount of CL. Additionally, tissues with different concentrations of hemoglobin will also have different refractive indexes. The CL intensity quantity and imaging resolution decrease with increasing tissue depth. Thus, the quantification ability of CL is relative poor especially for deep tissues and strongly depends on the tissue type and the location of the body. The major challenge for implementing CR-based imaging and detection technique is the variety and limited penetrability of light into deep tissue. However, this limitation can be minimized in small animal imaging, and CL also can be used as a depth-independent self-generating light source.

\section{ADVANTAGES AND POTENTIAL APPLICATIONS OF CLI}

In the past a few decades, interest in CR was mainly focused on the Cerenkov detector, which was used to monitor some radionuclides that do not emit gamma rays. Presently, it has been shown to be useful in imaging the in vivo distribution of radioactive probes, which are usually imaged by nuclear imaging modalities. In comparison to PET and SPECT, CLI is advantageous in terms of its low cost and high-throughput potential. As shown in some studies, CLI has possibility to image up to five animals in less than $5 \mathrm{~min}$, compared with PET scans, which require 5-20 min or longer to image one animal. Additionally, because CLI correlates highly with PET studies for superficial tissues, it has been used to quantitatively measure radiotracer uptake in vivo. With its high throughput and resolution, CLI is beneficial for imaging and analysis, especially for some superficial tumors or organs.

However, the application of CLI is limited by its low tissue penetration ability, as discussed in the previous sections. This restricts the use of CLI to small animal research or to human superficial tissues. Some studies have demonstrated that CL could serve as an alternative internal excitation source by using energy transfer strategies, which opens new directions for CLI. CLI thus can be improved through excitation fluorophores to produce NIR light, which is more suitable for in vivo OI, or it could be combined with PDT to increase the efficacy of treatments. As reported by several studies, some photosensitizers for PDT need to be excited by UV or blue light, which almost match the CL spectrum $[84,85]$. Therefore, CL could be used to excite such molecules for PDT, especially when combined with therapeutic radionuclides. 
This strategy may supply potential clinical benefits not only for superficial tumors but also for deep tumors. Additionally, the high-energy X-rays or electrons used for radiotherapy can also induce CL, which could be used for activating photosensitizers for PDT. This type of strategy would combine radiotherapy and PDT, and it may also monitor the radiation dose accumulation by CLI.

\section{CONCLUSION}

The CR provides a simple, quantitative tool for radionuclide detection, CLI, radiotherapy monitoring, endoscopy, and optical-guided surgery and for the excitation of fluorophores or other photosensitizers. With further development, it may benefit researchers by serving as a novel multi-functional tool for biology research.

\section{ACKNOWLEDGMENTS}

This work was supported, in part, by the Office of Science (BER), U.S. Department of Energy (DE-SC0008397), NCI In vivo Cellular Molecular Imaging Center (ICMIC) grant P50 CA114747, and the National Natural Science Foundation of China (81230033).

\section{REFERENCES}

1. Xu Y, Liu H, Cheng Z. Harnessing the power of radionuclides for optical imaging: cerenkov luminescence imaging. J Nucl Med. (2011) 52:2009-18. doi: 10.2967/jnumed.111.092965

2. Thorek D, Robertson R, Bacchus WA, Hahn J, Rothberg J, Beattie BJ, et al. Cerenkov imaging - a new modality for molecular imaging. Am J Nucl Med Mol Imaging (2012) 2:163-73.

3. Spinelli AE, Marengo M, Calandrino R, Sbarbati A, Boschi F. Optical imaging of radioisotopes: a novel multimodal approach to molecular imaging. Q $\mathrm{Nucl}$ Med Mol Imaging J. (2012) 56:280-90.

4. Mitchell GS, Gill RK, Boucher DL, Li C, Cherry SR. In vivo Cerenkov luminescence imaging: a new tool for molecular imaging. Philos Trans Math Phys Eng Sci A (2011) 369:4605-19. doi: 10.1098/rsta.2011.0271

5. Lucignani G. Cerenkov radioactive optical imaging: a promising new strategy. Eur Nucl Med Mol Imaging J. (2011) 38:592-5. doi: 10.1007/s00259-0101708-6

6. Chin PT, Welling MM, Meskers SC, Valdes Olmos RA, Tanke H, van Leeuwen FW. Optical imaging as an expansion of nuclear medicine: cerenkov-based luminescence vs fluorescence-based luminescence. Eur Nucl Med Mol Imaging J. (2013). 40:1282-91. doi: 10.1007/s00259-013-2408-2409

7. Jelley JV. Cerenkov radiation and its applications. Br J Appl Phys. (1955) 6:227-32.

8. Spinelli AE, D’Ambrosio D, Calderan L, Marengo M, Sbarbati A, Boschi F. Cerenkov radiation allows in vivo optical imaging of positron emitting radiotracers. Phys Med Biol. (2010) 55:483-95. doi: 10.1088/0031-9155/55/2/010

9. Mitchell GS, Gill RK, Cherry SR. Comments on "Cerenkov radiation allows in vivo optical imaging of positron emitting radiotracers." Phys Med Biol. (2010) 55:L43-4. author reply: L45-9. doi: 10.1088/0031-9155/55/18/L01

10. Spinelli AE, D'Ambrosio D, Calderan L, Marengo M, Sbarbati A, Boschi F. Reply to "Comments on 'Cerenkov radiation allows in vivo optical imaging of positron emitting radiotracers.”' Phys Med Biol. (2010) 55:L45-9. doi: 10.1088/0031-9155/55/18/102

11. Beattie BJ, Thorek DL, Schmidtlein CR, Pentlow KS, Humm JL, Hielscher AH. Quantitative modeling of Cerenkov light production efficiency from medical radionuclides. PLoS ONE (2012) 7:e31402. doi: 10.1371/journal.pone.0031402

12. Ackerman NL, Graves EE. The potential for Cerenkov luminescence imaging of alpha-emitting radionuclides. Phys Med Biol. (2012) 57:771-83. doi: 10.1088/0031-9155/57/3/771

13. Liu H, Ren G, Miao Z, Zhang X, Tang X, Han P, et al. Molecular optical imaging with radioactive probes. PLoS ONE (2010) 5:e9470. doi: 10.1371/journal.pone.0009470
14. Cerenkov PA. Radiation of particles moveing at a velocity exceeding that of light, and some of the possibilities for their use in experimental physics. Nobel Lect. (1958).

15. Schaumloffel E, Pantke H, Graul EH. [Contribution to the determination of P32 activity by means of the Cerenkov effect in quantitative studies of the absorption of phosphate ions into the dentin]. Dtsch Zahnarztl Z (1967) 22:675-80.

16. Arinc A, Johansson LC, Gilligan CR, Pearce AK. Standardisation of (210) $\mathrm{Pb}$ by Cerenkov counting. Appl Radiat Isot. (2011) 69:768-72. doi: 10.1016/j.apradiso.2011.01.007

17. Parker RP, Elrick RH. The assay of beta-emitting radioisotopes using Cerenkov counting. Int Appl Radiat Isot J. (1966) 17:361-2.

18. Elrick RH, Parker RP. The use of Cerenkov radiation in the measurement of beta-emitting radionuclides. Int Appl Radiat Isot J. (1968) 19:263-71.

19. Francois B. Detection of hard beta-emitting radionuclides in aqueous solutions using Cerenkov radiation: a review article. Int Nucl Med Biol J. (1973) 1:1-14.

20. Gunther K, Lange S, Veit M. A rapid method for determining 89Sr and $90 \mathrm{Sr}$ by Cerenkov counting. Appl Radiat Isot. (2009) 67:781-5. doi: 10.1016/j.apradiso.2009.01.035

21. Lauchli A. Radioassay for beta-emitters in biological materials using Cerenkov radiation. Int Appl Radiat Isot J. (1969) 20:265-70.

22. Burch WM. Cerenkov light from $32 \mathrm{P}$ as an aid to diagnosis of eye tumours. Nature (1971) 234:358.

23. Smith RD, Anderson JJ, Ristic M, Huxsoll DL. The use of Cerenkov radiation in $32 \mathrm{P}$ labeled platelet survival studies. Int Appl Radiat Isot J. (1972) 23:513-7.

24. Robertson R, Germanos MS, Li C, Mitchell GS, Cherry SR, Silva MD. Optical imaging of Cerenkov light generation from positron-emitting radiotracers. Phys Med Biol. (2009) 54:N355-65. doi: 10.1088/0031-9155/54/16/N01

25. Cho JS, Taschereau R, Olma S, Liu K, Chen YC, Shen CK, et al. Cerenkov radiation imaging as a method for quantitative measurements of beta particles in a microfluidic chip. Phys Med Biol. (2009) 54:6757-71. doi: 10.1088/00319155/54/22/001

26. Ruggiero A, Holland JP, Lewis JS, Grimm J. Cerenkov luminescence imaging of medical isotopes. Nucl Med J. (2010) 51:1123-30. doi: 10.2967/jnumed.110.076521

27. Lewis MA, Kodibagkar VD, Oz OK, Mason RP. On the potential for molecular imaging with Cerenkov luminescence. Opt Lett. (2010) 35:3889-91. doi: 10.1364/OL.35.003889

28. Weissleder R. Molecular imaging in cancer. Science (2006) 312:1168-71. doi: 10.1126/science.1125949

29. Boschi F, Calderan L, D’Ambrosio D, Marengo M, Fenzi A, Calandrino R, et al. In vivo (1)(8)F-FDG tumour uptake measurements in small animals using Cerenkov radiation. Eur Nucl Med Mol Imaging J. (2011) 38:120-7. doi: 10.1007/s00259-010-1630-y

30. Zhang X, Kuo C, Moore A, Ran C. In Vivo optical imaging of interscapular brown adipose tissue with (18)F-FDG via cerenkov luminescence imaging. PLoS ONE (2013) 8:e62007. doi: 10.1371/journal.pone.0062007

31. Liu H, Ren G, Liu S, Zhang X, Chen L, Han P, et al. Optical imaging of reporter gene expression using a positron-emission-tomography probe. J Biomed Opt. (2010) 15:060505 doi: 10.1117/1.3514659

32. Kim DH, Choe YS, Choi JY, Lee KH, Kim BT. Binding of 2-[18F]fluoroCP-118,954 to mouse acetylcholinesterase: microPET and ex vivo Cerenkov luminescence imaging studies. Nucl Med Biol. (2011) 38:541-7 doi: 10.1016/j.nucmedbio.2010.11.010

33. Jeong SY, Hwang MH, Kim JE, Kang S, Park JC, Yoo JJ, et al. Combined Cerenkov luminescence and nuclear imaging of radioiodine in the thyroid gland and thyroid cancer cells expressing sodium iodide symporter: initial feasibility study. Endocr J. (2011) 58:575-83. doi: 10.1507/endocrj. K11E-051

34. Holland JP, Normand G, Ruggiero A, Lewis JS, Grimm J. Intraoperative imaging of positron emission tomographic radiotracers using Cerenkov luminescence emissions. Mol Imaging (2011) 10:177-86.

35. Natarajan A, Habte F, Liu H, Sathirachinda A, Hu X, Cheng Z, et al. Evaluation of Zr-rituximab tracer by cerenkov luminescence imaging and correlation with PET in a humanized transgenic mouse model to image NHL. Mol Imaging Biol. (2013) 15:468-75. doi: 10.1007/s11307-013-0624-0

36. Zalutsky MR. Radionuclide therapy. in: Handbook of Nuclear Chemistry. Springer US Press (2011) 2179-209. doi: 10.1007/978-1-13674419-0720-2_46 
37. Klain M, Ricard M, Leboulleux S, Baudin E, Schlumberger M. Radioiodine therapy for papillary and follicular thyroid carcinoma. Eur J Nucl Med Mol Imaging (2002) 29(Suppl. 2), S479-85. doi: 10.1007/s00259-0020810-9

38. Yang W, Qin W, Hu Z, Suo Y, Zhao R, Ma X, et al. Comparison of Cerenkov luminescence imaging (CLI) and gamma camera imaging for visualization of let-7 expression in lung adenocarcinoma A549 Cells. Nucl Med Biol. (2012) 39:948-53 doi: 10.1016/j.nucmedbio.2012.05.004

39. Hu Z, Yang W, Ma X, Ma W, Qu X, Liang J, et al. Cerenkov luminescence tomography of aminopeptidase N (APN/CD13) expression in mice bearing HT1080 tumors. Mol Imaging (2013) 12:173-81.

40. Aweda TA, Eskandari V, Kukis DL, Boucher DL, Marquez BV, Beck HE, et al. New covalent capture probes for imaging and therapy, based on a combination of binding affinity and disulfide bond formation. Bioconjug Chem. (2011) 22:1479-83. doi: 10.1021/bc2002049

41. Park JC, Il An G, Park SI, Oh J, Kim HJ, Su Y, et al. Luminescence imaging using radionuclides: a potential application in molecular imaging. $\mathrm{Nucl} \mathrm{Med}$ Biol. (2011) 38:321-9. doi: 10.1016/j.nucmedbio.2010.09.003

42. Spinelli AE, Boschi F. Unsupervised analysis of small animal dynamic Cerenkov luminescence imaging. J Biomed Opt. (2011) 16:120506. doi: $10.1117 / 1.3663442$

43. Wang Y, Liu Y, Luehmann H, Xia X, Wan D, Cutler C, et al. Radioluminescent gold nanocages with controlled radioactivity for real-time in vivo imaging. Nano Lett. (2013) 13:581-5. doi: 10.1021/nl304111v

44. Xu Y, Chang E, Liu H, Jiang H, Gambhir SS, Cheng Z. Proof-of-concept study of monitoring cancer drug therapy with cerenkov luminescence imaging. J Nucl Med. (2012) 53:312-7. doi:10.2967/jnumed.111.094623

45. Xu Y, Liu H, Chang E, Jiang H, Cheng Z. Cerenkov Luminescence Imaging (CLI) for cancer therapy monitoring. J Vis Exp. (2012) e4341. doi: $10.3791 / 4341$

46. Robertson R, Germanos MS, Manfredi MG, Smith PG, Silva MD. Multimodal imaging with (18)F-FDG PET and Cerenkov luminescence imaging after MLN4924 treatment in a human lymphoma xenograft model. J Nucl Med. (2011) 52:1764-9. doi: 10.2967/jnumed.111.091710

47. Thorek DL, Abou DS, Beattie BJ, Bartlett RM, Huang R, Zanzonico PB. Positron lymphography: multimodal, high-resolution, dynamic mapping and resection of lymph nodes after intradermal injection of 18F-FDG. J Nucl Med. (2012) 53:1438-45. doi: 10.2967/jnumed.112.104349

48. Zhang JG, Liu HF. Functional imaging and endoscopy. World J Gastroenterol. (2011) 17:4277-82. doi: 10.3748/wjg.v17.i38.4277

49. Kothapalli SR, Liu H, Liao JC, Cheng Z, Gambhir SS. Endoscopic imaging of Cerenkov luminescence. Biomed Opt Exp. (2012) 3:1215-25. doi: 10.1364/BOE.3.001215

50. Liu H, Carpenter CM, Jiang H, Pratx G, Sun C, Buchin MP, et al. Intraoperative imaging of tumors using Cerenkov luminescence endoscopy: a feasibility experimental study. J Nucl Med. (2012) 53:1579-84. doi: 10.2967/jnumed.111.098541

51. Justus BL, Falkenstein P, Huston AL, Plazas MC, Ning H, Miller RW. Elimination of Cerenkov interference in a fibre-optic-coupled radiation dosemeter. Radiat Prot Dosimetry (2006) 120:20-3. doi: 10.1093/rpd/nci525

52. Lambert J, McKenzie DR, Law S, Elsey J, Suchowerska N. A plastic scintillation dosimeter for high dose rate brachytherapy. Phys Med Biol. (2006) 51:5505-16. doi: 10.1088/0031-9155/51/21/008

53. Lambert J, Yin Y, McKenzie DR, Law S, Suchowerska N. Cerenkov-free scintillation dosimetry in external beam radiotherapy with an air core light guide. Phys Med Biol. (2008) 53:3071-80. doi: 10.1088/0031-9155/53/11/021

54. Liu PZ, Suchowerska N, Abolfathi P, McKenzie DR. Real-time scintillation array dosimetry for radiotherapy: the advantages of photomultiplier detectors. Med Phys. (2012) 39:1688-95. doi: 10.1118/1.3690465

55. Liu PZ, Suchowerska N, Lambert J, Abolfathi P, McKenzie DR. Plastic scintillation dosimetry: comparison of three solutions for the Cerenkov challenge. Phys Med Biol. (2011) 56:5805-21. doi: 10.1088/0031-9155/56/18/003

56. Jang KW, Yoo WJ, Shin SH, Shin D, Lee B. Fiber-optic Cerenkov radiation sensor for proton therapy dosimetry. Opt Exp. (2012) 20:13907-14. doi: 10.1364/OE.20.013907

57. Jang KW, Yagi $\mathrm{T}$, Pyeon $\mathrm{CH}$, Yoo WJ, Shin $\mathrm{SH}$, Jeong $\mathrm{CB}$, et al. Application of Cerenkov radiation generated in plastic optical fibers for therapeutic photon beam dosimetry. J Biomed Opt. (2013) 18:27001. doi: 10.1117/1.JBO.18.2.027001
58. Glaser AK, Davis SC, Voigt WH, Zhang R, Pogue BW, Gladstone DJ. Projection imaging of photon beams using Cerenkov-excited fluorescence. Phys Med Biol. (2013) 58:601-19. doi: 10.1088/0031-9155/58/ $3 / 601$

59. Axelsson J, Davis SC, Gladstone DJ, Pogue BW. Cerenkov emission induced by external beam radiation stimulates molecular fluorescence. Med Phys. (2011) 38:4127-32. doi: 10.1118/1.3592646

60. Vaupel P, Mayer A, Hockel M. Impact of hemoglobin levels on tumor oxygenation: the higher, the better? Strahlenther Onkol. (2006) 182:63-71. doi: 10.1007/s00066-006-1543-7

61. Axelsson J, Glaser AK, Gladstone DJ, Pogue BW. Quantitative Cherenkov emission spectroscopy for tissue oxygenation assessment. Opt Exp. (2012) 20:5133-42. doi: 10.1364/OE.20.005133

62. Zhang R, Glaser A, Esipova TV, Kanick SC, Davis SC, Vinogradov SD et al. Cerenkov radiation emission and excited luminescence (CREL) sensitivity during external beam radiation therapy: monte Carlo and tissue oxygenation phantom studies. Biomed Opt Exp. (2012) 3:2381-94. doi: 10.1364/BOE.3.002381

63. Zhang R, Davis SC, Demers JL. Glaser AK, Gladstone DJ, Esipova TV et al. Oxygen tomography by Cerenkov-excited phosphorescence during external beam irradiation. J Biomed Opt. (2013) 18:50503. doi: 10.1117/1.JBO.18.5.050503

64. Glaser AK, Voigt WH, Davis SC, Zhang R, Gladstone DJ, Pogue BW. Three-dimensional Cerenkov tomography of energy deposition from ionizing radiation beams. Opt Lett. (2013) 38:634-6. doi: 10.1364/OL.38. 000634

65. Liu H, Zhang X, Xing B, Han P, Gambhir SS, Cheng Z. Radiationluminescence-excited quantum dots for in vivo multiplexed optical imaging. Small (2010) 6:1087-91. doi: 10.1002/smll.200902408

66. Carpenter CM, Sun C, Pratx G, Liu H, Cheng Z, Xing L. Radioluminescent nanophosphors enable multiplexed small-animal imaging. Opt Express (2012) 20:11598-604. doi: 10.1364/OE.20.011598

67. Ran C, Zhang Z, Hooker J, Moore A. In vivo photoactivation without "light": use of Cherenkov radiation to overcome the penetration limit of light. Mol Imaging Biol. (2012) 14:156-62. doi: 10.1007/s11307-011-0489-z

68. Thorek DL, Ogirala A, Beattie BJ, Grimm J. Quantitative imaging of disease signatures through radioactive decay signal conversion. Nat Med. (2013). 52:7756-60. doi: 10.1038/nm.3323

69. Kotagiri N, Niedzwiedzki DM, Ohara K, Achilefu S. Activatable probes based on distance-dependent luminescence associated with cerenkov radiation. Angew Chem Int Ed Engl. (2013). 18:20502. doi: 10.1002/anie.201302564

70. Spinelli AE, Ferdeghini M, Cavedon C, Zivelonghi E, Calandrino R, Fenzi AA, et al. First human Cerenkography. J Biomed Opt. (2013) 18:20502. doi: 10.1117/1.JBO.18.2.020502

71. Thorek DL. Riedl C, Grimm J. Clinical Cerenkov Luminescence Imaging of 18F-FDG. J Nucl Med. (2013). 5:e13300. doi: 10.2967/jnumed.113.127266

72. Weissleder R. A. clearer vision for in vivo imaging. Nat Biotechnol. (2001) 19:316-7. doi: 10.1038/86684

73. Dothager RS, Goiffon RJ, Jackson E, Harpstrite S, Piwnica-Worms D. Cerenkov radiation energy transfer (CRET) imaging: a novel method for optical imaging of PET isotopes in biological systems. PLoS ONE (2010) 5:e13300. doi: 10.1371/journal.pone. 0013300

74. Spinelli AE, Boschi F. Optimizing in vivo small animal Cerenkov luminescence imaging. J Biomed Opt. (2012) 17:040506. doi: 10.1117/1.JBO.17.4.040506

75. Li C, Mitchell GS, Cherry SR. Cerenkov luminescence tomography for small-animal imaging. Opt Lett. (2010) 35:1109-11. doi: 10.1364/OL.35. 001109

76. Chaudhari AJ, Darvas F, Bading JR, Moats RA, Conti PS, Smith DJ, et al. Hyperspectral and multispectral bioluminescence optical tomography for small animal imaging. Phys Med Biol. (2005) 50:5421-41. doi: 10.1088/00319155/50/23/001

77. Spinelli AE, Kuo C, Rice BW, Calandrino R, Marzola P, Sbarbati A, et al. Multispectral Cerenkov luminescence tomography for small animal optical imaging. Opt Express (2011) 19:12605-18. doi: 10.1364/OE.19. 012605

78. Hu Z, Liang J, Yang W, Fan W, Li C, Ma XX, et al. Experimental Cerenkov luminescence tomography of the mouse model with SPECT imaging validation. Opt Express (2010) 18:24441-50. doi: 10.1364/OE.18. 024441 
79. Hu Z, Ma X, Qu X, Yang W, Liang J, Wang J, et al. Three-dimensional noninvasive monitoring iodine-131 uptake in the thyroid using a modified Cerenkov luminescence tomography approach. PLoS ONE (2012) 7:e37623. doi: 10.1371/journal.pone.0037623

80. Hu Z, Chen X, Liang J, Qu X, Chen D, Yang WJ, et al. Single photon emission computed tomography-guided Cerenkov luminescence tomography. J Appl Phys. (2012) 112:024703. doi: 10.1063/1.4739266

81. Zhong J, Tian J, Yang X, Qin C. L1-regularized Cerenkov luminescence tomography with a SP3 method and CT fusion. Conf Proc IEEE Eng Med Biol Soc. (2011) 2011:6158-61. doi: 10.1109/IEMBS.2011.6091521

82. Zhong J, Qin C, Yang X, Chen Z, Yang X, Tian J. Fast-specific tomography imaging via Cerenkov emission. Mol Imaging Biol. (2012) 14:286-92. doi: 10.1007/s11307-011-0510-6

83. Zhong J, Tian J, Yang X, Qin C. Whole-body Cerenkov luminescence tomography with the finite element SP(3) method. Ann Biomed Eng. (2011) 39:1728-35. doi: 10.1007/s10439-011-0261-1

84. Felgentrager A, Gonzales FP, Maisch T, Baumler W. Ion-induced stacking of photosensitizer molecules can remarkably affect the luminescence detection of singlet oxygen in Candida albicans cells. J Biomed Opt. (2013) 18:045002. doi: 10.1117/1.JBO.18.4.045002
85. Nowak-Stepniowska A, Pergol P, Padzik-Graczyk A. Photodynamic method of cancer diagnosis and therapy-mechanisms and applications. Postepy Biochem. (2013) 59:53-63.

Conflict of Interest Statement: The authors declare that the research was conducted in the absence of any commercial or financial relationships that could be construed as a potential conflict of interest.

Received: 25 September 2013; accepted: 14 January 2014; published online: 03 February 2014.

Citation: Ma X, Wang J and Cheng Z (2014) Cerenkov radiation: a multi-functional approach for biological sciences. Front. Physics 2:4. doi: 10.3389/fphy.2014.00004

This article was submitted to Biomedical Physics, a section of the journal Frontiers in Physics.

Copyright (c) $2014 \mathrm{Ma}$, Wang and Cheng. This is an open-access article distributed under the terms of the Creative Commons Attribution License (CC BY). The use, distribution or reproduction in other forums is permitted, provided the original author(s) or licensor are credited and that the original publication in this journal is cited, in accordance with accepted academic practice. No use, distribution or reproduction is permitted which does not comply with these terms. 